\title{
Réplique au compte rendu de Lucia Ferretti du livre de Luca Codignola et Luigi Bruti-Liberati, intitulé Storia del Canada, dalle origini ai giorni nostri, paru dans le volume 53,3 (hiver 2000) : 429-433.
}

\section{Luigi Bruti-Liberati et Luca Codignola}

Volume 53, numéro 4, printemps 2000

Histoire des Premières Nations : nouvelles lectures et nouveaux

problèmes

URI : https://id.erudit.org/iderudit/005414ar

DOI : https://doi.org/10.7202/005414ar

Aller au sommaire du numéro

Éditeur(s)

Institut d'histoire de l'Amérique française

ISSN

0035-2357 (imprimé)

1492-1383 (numérique)

Découvrir la revue

Citer ce compte rendu

Bruti-Liberati, L. \& Codignola, L. (2000). Compte rendu de [Réplique au compte rendu de Lucia Ferretti du livre de Luca Codignola et Luigi Bruti-Liberati, intitulé Storia del Canada, dalle origini ai giorni nostri, paru dans le volume 53,3 (hiver 2000) : 429-433.] Revue d'histoire de l'Amérique française, 53(4), 631-635. https://doi.org/10.7202/005414ar 
Réplique au compte rendu de Lucia Ferretti du livre de Luca Codignola et Luigi BrutiLiberati, intitulé Storia del Canada, dalle origini ai giorni nostri, paru dans le volume 53,3 (hiver 2000):429-433.

Nous nous réjouissons du fait que la plus importante revue du monde consacrée à l'histoire de l'Amérique française ait pris la décision de faire rédiger un véritable compte rendu, et non pas une simple note bibliographique, de notre livre. Nous sommes aussi très reconnaissants envers Lucia Ferretti qui a consacré autant d'espace à examiner nos positions. Toutefois, nous sommes surpris par la violence de ses critiques, d'autant plus que nulle part, dans son compte rendu, elle n'indique à ses lecteurs les omissions, les imprécisions ou les erreurs que nous aurions commises Notre Storia del Canada étant en italien, nous croyons qu'une réplique peut être utile aux lecteurs de la RHAF pour mieux resituer notre synthèse à l'intérieur du débat historiographique en cours dans la communauté scientifique internationale.

Madame Ferretti s'en prend surtout à l'un des deux auteurs, Luca Codignola, qu'elle accuse de «se livre[r] à un remaniement si considérable de la mémoire historique des Canadiens que l'entreprise paraît obéir à des fins politiques plus que scientifiques». Nous avouons que, bien que nous ayons écrit et signé séparément nos parties et que nous ne nions pas les différences de détail, nous en partageons les principes inspirateurs et le cadre interprétatif. II nous paraît donc assez étrange que Ferretti juge le propos des deux auteurs, de même que leur intégrité et leur honnêteté intellectuelle, de façon si différente.

Lucia Ferretti se débarrasse de la deuxième partie de notre livre, portant sur la période allant de 1867 à 1999 (p 447-727) et signée par Luigi Bruti-Liberati, en la qualifiant de «synthèse honnête» bien que «somme toute classique», suffisante peut-être pour «un public non spécialiste, Italiens d'Italie et Italiens d'ici», mais certainement pas en mesure d'apprendre «beaucoup» aux «canadianistes». Elle est encore plus 
sévère envers la première partie, des origines à 1867 (p. 11-446), signée par Codignola, qu'elle traite à la fin de son compte rendu et définit comme «profondement déplaisant[e]» à cause de son «côté d'histoire à l'eau de rose», de son traitement de «la question des "francophones"», et surtout de son «remaniement [...] de la mémoire historique des Canadiens». Faisant semblant d'oublier que notre Storia del Canada concerne I'histoire d'un espace géographique bien vaste, Ferretti choisit de l'examiner comme si notre livre était une histoire de la nation canadiennefrançaise. Dès lors, elle ne dit pas un seul mot des autres provinces, elle évoque très rapidement les États-Unis et les Autochtones, et, surtout, elle ne semble pas avoir perçu la «perspective européenne», qui, selon nous, rend notre livre différent des histoires du Canada écrites par les historiens nord-américains

Dans quelques cas, peu nombreux d'ailleurs, Lucia Ferretti prétend faire des corrections que nous attribuons à une lecture insuffisamment approfondie et à une utilisation hors contexte de certaines affirmations. N ous nous limiterons à deux exemples. Premièrement, elle se scandalise du fait que Jacques Cartier ne soit «mentionné [qu']en note infrapaginale» (p. 41, note 14). Elle ne semble pas avoir vu que deux pages plus loin (p. 43-44), nous consacrons quelque 260 mots à décrie l'«intérêt tout spécial » de ses relations avec les Autochtones, le cadre idéologique et juridique de la présence française qu'il contribue à créer, et I'«importance énorme» des écrits qui lui sont attribués. Deuxièmement, nous n'écrivons pas qu'une majorité des Acadiens «parle micmac à la maison », mais tout simplement que la famille de Philippe d'Azit, dit Meuse, le faisait à Merliguesh à la fin du xviI ${ }^{\mathrm{e}}$ siècle (p. 47).

Parfois, la veine polémique pousse Ferretti trop loin. Elle reproche ainsi à Codignola d'avoir décrit «les francophones du Québec [...] sous le Régime français [...] essentiellement à partir du témoignage que le gouverneur James Murray a laissé d'eux», de sorte que le lecteur est amené à croire que l'auteur, qui n'aurait pas consulté d'autres sources, partage le point de vue du fonctionnaire britannique. Or, Codignola n'a retenu I'analyse de Murray, qu'il juge très pénétrante, que comme point de départ pour un examen en profondeur de la société canadienne. Lucia Ferretti reproche aussi à Codignola d'avoir écrit que «le statut» de l'Église catholique était «meilleur après qu'avant» la Conquête. Là encore, Codignola ne fait que rapporter le point de vue de l'évêque de Q uébec, Jean-Olivier Briand, et de son vicaire général, Pierre de La Rue, l'abbé de L'Îsle-Dieu, surpris devant le fait que la catastrophe ne s'était 
pas produite telle qu'attendue. Dans les deux cas Codignola ne fait que partager le principe professionnel qui dit que l'historien doit examiner le passé à travers les yeux des gens du passé, tout en tirant profit de l'énorme avantage de connaître leur avenir pour ainsi rendre leur histoire dans sa complexité la plus extensive possible.

D'autres accusations sont de caractère plus général. Par exemple, Lucia Ferretti affirme que les femmes «sont très peu présentes dans cette Storia » et que Bruti-Liberati réserve une place «assez mince» à I'histoire sociale. À ce propos, nous aurions aimé que Ferretti fournisse quelques exemples spécifiques. En outre, en dépit d'une bibliographie commentée de 35 pages composée presque entièrement d'ouvrages parus pendant les années 1990, Lucia Ferretti nous accuse de nous être surtout inspirés des auteurs anglophones (William J. Eccles et les tandems David J. Bercuson-Jack L. Granatstein et John A. Dickinson-Brian Young), dont les ouvrages, à la différence de «l'historiographie rédigée en français» qui sert [...] seulement à documenter des questions spécifiques», auraient le vice de nous avoir inspiré la manière de poser «les problèmes généraux». Bien qu'en désaccord, nous admettons volontiers qu'un compte rendu d'une synthèse ne peut pas s'attarder à tous les détails, non plus que la synthèse elle-même par ailleurs. En outre, les commentaires de ce genre font partie du domaine du débat scientifique légitime. Chaque historienne ou historien choisit sa perspective et ses éléments à partir d'une grille d'interprétation qui n'est pas nécessairement celle du recenseur.

L'essentiel de la critique de Ferretti concerne Codignola, qui serait davantage coupable d' «obéir à des fins politiques plus que scientifiques». En effet, en soulignant dès le début que notre ouvrage a été «subventionné par le ministère des Affaires extérieures du Canada», elle invite le lecteur à traiter notre livre comme un pamphlet politique de 815 pages et ses auteurs comme des agents du gouvemement fédéral qui auraient un «parti pris trop visible [...] pour la bonne-entente». Étant donné que la plupart des historiens professionnels, québécois, canadiens et autres, reçoivent des subventions, nous nous demandons si l'on est en train de proposer l'institution d'un bureau qui questionnerait l'intégrité personnelle des auteurs, avant de consentir aux pairs une critique scientifique de leurs ouvrages. Que dirait-on d'un ouvrage subventionné de quelque sorte par le ministère des Relations internationales du Québec, le Conseil international d'études canadiennes ou I'Association intemationale d'études québécoises? 
Qu'est-ce que Codignola aurait fait pour «obéir» à ces «fins politiques»? Selon Ferretti, il se serait donné trois objectifs. Premièrement, il aurait montré que, depuis l'arrivée des premiers Autochtones, le Canada a été un pays d'immigrants et une société multiculturelle. Nous confirmons l'usage du premier propos. Pour ce qui est de la société multiculturelle, Codignola n'utilise le mot qu'une seule fois dans la période après la Conquête (pour nier son existence [p 328]), et Bruti-Liberati ne traite du multiculturalisme qu'en tant que politique du gouvernement fédéral (p. 709-712).

Deuxièmement, Codignola aurait essayé d'«ôter toute légitimité à la prétention des Canadiens français de former une nation». Bien au contraire. Nous croyons que les Canadiens français forment depuis longtemps une société, une communauté, un pays, un peuple ou une nation - peu importe le mot - avec une conscience de leur identité bien distincte et aussi le droit à la souveraineté. (Nous avons bien mis en évidence le prix en vies humaines payé par les États-Unis au xix ${ }^{e}$ siècle pour avoir nié ce droit aux États de la Confédération.) Mais nous ne croyons pas que cela implique l'absence de diférences et même de contrastes majeurs au sein de cette nation. II nous paraît donc paradoxal qu'une historienne de la société et de la religion telle que Ferretti nous accuse d'avoir analysé la société canadienne-française après la Conquête selon les «groupes sociaux entre lesquels ils se séparent» plutôt que comme «un peuple».

Troisièmement, Codignola aurait montré comment pendant la première moitié $d u x{ }^{e}$ siècle les exigences de la collaboration à l'échelle continentale auraient été plus fortes que les régionalismes potentiellement centrifuges, d'où la Confédération. Nous confirmons ce propos. Nous croyons que le travail de l'historien consiste à connaître les options des gens du passé, à raconter et à expliquer comment les décisions prises par chacune et chacun ont contribué à modifier leurs vies, individuelles et collectives. II nous paraît évident que les gens du passé, que la chose plaise ou pas, ont choisi jusqu'à présent de former une seule Confédération. Cela n'implique pas I'unanimité et I'harmonie «à l'eau de rose» mais, au contraire, la dissonance continuelle, les équilibres provisoires, les ambiguïtés et les ambivalences, la nécessité pour les communautés de partager le même territoire, tout comme la recherche constante de solutions qui permettent la vie en commun. En effet, nous croyons qu'il n'appartient pas aux historiens de juger les gens du passé par apport à un projet politique qui conceme le futur d'une société, que nous le partagions ou pas. 
Lucia Ferretti reproche à Codignola d'avoir essayé d'atteindre ces objectifs à travers un «remaniement [...] considérable de la mémoire historique des Canadiens». Par exemple, aucune des «dates charnières de l'histoire de la N ouvelle-France» (1534-1535, 1608, 1759-1760) naurait été retenue. En effet, «[l]es chronologies ne sont pas neutres», affirmait J. Létourneau en 1997, et Dickinson et Young avaient posé la question dès 1991. Est-ce que Ferretti croit vraiment que le grand récit accrédité ( «la mémoire historique» de grands événements dépresseurs) serait forcément unique? Pourtant, en changeant de récit, il est bien évident que I'on change de balises Par exemple, c'est à cause de la nouvelle importance que nous donnons aux Autochtones que nous arons choisi l'année de la Paix de Montréal (1701) comme date limite des chapitres 2 et 3 . Et c'est à cause du grand remaniement de l'Empire britannique de la fin du xvIII ${ }^{e}$ siècle que nous arons placé la Conquête (dont l'importance fondatrice justifie notre définition de «révolution canadienne») à l'intérieur d'un chapitre qui commence en 1744 et se temine en 1791.

En conclusion, notre impression est que Lucia Ferretti aurait dû envoyer son compte rendu à une revue de politique ou à un quotidien plutôt qu'à une revue scientifique telle que la RHAF. Par ailleurs, une traduction en anglais de notre livre est en cours. N ous espérons qu'une traduction en français se fasse rapidement pour permettre aux lecteurs francophones de l'Amérique du Nord de partager avec nous la conviction qu'une vision de leur histoire, placée comme elle le mérite dans le cadre du monde occidental, lui donne toute l'importance et la richesse qu'elle possède.

LUIGI BRUTI-LIBERATI Università di Milano

LUCA CODIGNOLA Università di Genova

Réponse

Je suis surprise du ton de la réplique de mes deux collègues J'invite les lecteurs à relire le compte rendu que j'ai préparé de leur ouvrage, et à juger par eux-mêmes du ton que j'ai moi-même employé. Par ailleurs, je maintiens mon propos intégralement. 